Methods With various levels of depression, as according to the Centre for Epidemiological Studies - Depression (CES-D) scale, 88 HIV newly diagnosed subjects were enrolled in the study. After 6-month intervention of case management, their depression status were re-evaluated and compared.

Results No significant differences for depression status were found 6 months after the intervention of case management among study subjects $(\mathrm{p}=0.345)$. However, the results of linear regression analysis indicated that those who had family support (beta $=0.303, p=0.012$ ) and lower HIV viral load (beta $=$ -0.265, $p=0.041$ ) would have better improvement for depression.

Conclusion Our findings indicate case management offers no apparent help lowering depression among PLWHA. However, HIV case managers should remind and assist physicians to paying more attention to PLWHA with lower family support or higher HIV viral load to avoid depression symptoms happened. Disclosure of interest statement The Australasian Society for HIV Medicine recognises the considerable contribution that industry partners make to professional and research activities. We also recognise the need for transparency of disclosure of potential conflicts of interest by acknowledging these relationships in publications and presentations. Nothing to declare.

\section{P18.12 PEOPLE SEEKING SEXUALLY TRANSMITTED DISEASES SCREENING, MEDICAL PROFESSIONALS, AND MEMBERS OF THE GENERAL PUBLIC SURVEYED REGARDING KNOWLEDGE ABOUT NON-OCCUPATIONAL POST- EXPOSURE PROPHYLAXIS FOR HIV}

${ }^{1} \mathrm{MC}$ Feng ${ }^{*},{ }^{1} \mathrm{CT} \mathrm{Yu},{ }^{2} \mathrm{ZW}$ Liang, ${ }^{1} \mathrm{SF}$ Liu, ${ }^{1} \mathrm{YC}$ Chuang, ${ }^{3} \mathrm{PL}$ Lu. ${ }^{1} \mathrm{Kaohsiung}$ Medical University Hospital, Kaohsiung Medical University, Nursing, Kaohsiung, Taiwan, Province of China; ${ }^{2}$ Kaohsiung Medical University, Kaohsiung, Taiwan, Province of China; ${ }^{3}$ Kaohsiung Medical University Hospital, Kaohsiung Medical University, Internal Medicine, Kaohsiung, Taiwan, Province of China

10.1136/sextrans-2015-052270.635

Introduction Non-occupational post-exposure prophylaxis (NPEP), a medical intervention to prevent HIV infection from risk behaviours conducted by general public, has been advocated by the Centre for Disease Control since 2013. However, general awareness has not yet risen. The aim of this study was to evaluate the knowledge of NPEP among subjects seeking for anonymous screening for sexually transmitted diseases (STD), healthcare professionals and general population in Taiwan.

Methods A cross sectional study was conducted with a structured questionnaire for 200 subjects who sought for anonymous STD screening, 125 healthcare professionals who worked in the departments of emergency and infectious diseases and 200 subjects who were recruited from the community to participate in the study. The questionnaire contains 10 items, which embedded NPEP-related knowledge. Statistical analysis was performed on the responses so as to ascertain the knowledge level of NPEP across the different groups.

Results Only 56\% of those receiving anonymous STD screening and $34 \%$ of the general population group have ever heard of NPEP, while $83.2 \%$ of healthcare professionals have heard of NPEP. A significantly higher proportion of healthcare professionals was found to know about NPEP $(\mathrm{p}<0.001)$. Overall, the response rate for correct answers for NPEP-related knowledge was found to be significantly higher for the subjects seeking for anonymous STD screening (24.1\%; healthcare professionals, $2.9 \%$; general population, $0 \%$; $\mathrm{p}<0.001$ ).

Conclusion Our study results indicate that over $80 \%$ of healthcare professionals know about NPEP, however without correct knowledge. Those who seek for anonymous STD screening have a better understanding of NPEP. Efforts should be reinforced to raise the NPEP awareness.

Disclosure of interest statement The Australasian Society for HIV Medicine recognises the considerable contribution that industry partners make to professional and research activities. We also recognise the need for transparency of disclosure of potential conflicts of interest by acknowledging these relationships in publications and presentations. Nothing to declare.

\section{P18.13 REPORTED CHURCH ATTENDANCE AT THE TIME OF ENTRY INTO HIV CARE ASSOCIATES WITH REDUCED VIREMIA AT 12 MONTHS}

N Van Wagoner, L Elopre*, AO Westfall, M Mugavero, J Turran, E Hook. Division of Infectious Diseases, Department of Medicine, University of Alabama at Birmingham, Birmingham AL

\subsection{6/sextrans-2015-052270.636}

Background The Southeastern US is characterised as America's "bible belt" with prominent religiosity yet also has the highest HIV incidence. The interplay between religion/spirituality and HIV-related outcomes could vary from detrimental to beneficial. We previously showed that men who have sex with men (MSM) who attended church were more likely to present to care with more advanced disease than those who did not. Here, we evaluate the relationship between church attendance and sustained HIV viremia 12 months after initiation of care in the Southeastern US.

Methods We evaluated 12-month longitudinal analysis was performed to evaluate the relationship between church attendance and continued viremia (viral load > 200 copies $/ \mathrm{ml}$ ) for patients presenting for care at a university HIV clinic. Univariate and multivariable logistic regression models were fit for church attendance (the main variable of interest) as well as other variables potentially related to sustained viremia.

Results Between 2007 to 2012, 382 patients initiated HIV care for the first time and had a HIV viral load available 12 months from time of care entry. Most were black (60\%), MSM (65\%) and at 12 months were virally suppressed (74\%). In MV analyses, reported church attendance was associated with a lower likelihood of HIV viremia (AOR 0.5; 95\% CI 0.2, 0.9). Variables associated with an increased likelihood of sustained HIV viremia included black race (AOR 3.2; 95\% CI 1.4, 7.4), living with family (AOR 2.7; 95\% CI 1.0, 6.9), and disclosure of HIV status only to family (AOR $3.0 ; 95 \%$ CI $1.2,7.7$ ) or only to friends (AOR 2.6; 95\% CI 1.1, 6.7).

Conclusions Church attendance may provide much needed support for patients entering HIV care. Further research is needed to understand the complex relationship between church attendance and health care outcomes in PLWH.

Disclosure of interest statement No disclosures. 